\title{
Prostatic Artery Embolisation with Bletilla Striata Particles in Treatment of Moderate to Severe Lower Urinary Tract Symp- toms (LUTS) Resulted from Benign Prostatic Hyperplasia (BPH)
}

\author{
Xuelu Zhou ${ }^{1^{*}}$, Shangjun Zhou ${ }^{2}$, Ping Zhan ${ }^{2}$ and Jianfeng Zhang ${ }^{2}$ \\ ${ }^{1}$ Department of Surgery, Chashan Hospital, Guangdong Medical University, China \\ ${ }^{2}$ Department of Surgery, Dongguan Hospital, Guangzhou University of Chinese Medicine, China
}

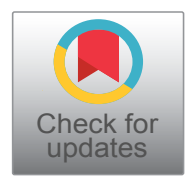

*Corresponding author: Dr. Xuelu Zhou, Department of Surgery, Chashan Hospital, Guangdong Medical University, 92 Caihong Road, Chashan Town, Dongguan, Guandong 523378, P.R. of China, Tel: 86-769-81378289, Fax: 86-769-86417066

\begin{abstract}
Background: The aim of this study was to explore the safety and efficacy of prostatic arterial embolization (PAE) with Bletilla striata particles as embolic agent in treatment of lower urinary tract symptoms (LUTS) resulted from benign prostatic hyperplasia (BPH)

Methods: Superselective angiogram of prostatic artery was performed with Seldinger's technique, and the artery was occluded with Bletilla striata particles 150-200 mm. PAE was successful in 68 of 75 patients $(90.7 \%)$.

Results: The total effective rate in international prostate symptoms score (IPSS), quality of life (QOL), peak urinary flow (Qmax), postvoid residual volume (PVR) at 1, 3, 6, 12 months was $100 \%$ (68/68), 100\% (68/68), 95.6\% (65/68), and $95.6 \%$ (65/68), respectively. At 12-month follow-up, the mean IPSS, QOL, and PVR decreased from $25.86 \pm 3.53$ to $6.52 \pm 1.51$ points $(P<0.01)$, from $4.56 \pm 0.61$ to $1.26 \pm 0.43$ points $(P<0.01)$, and from $131.52 \pm 19.13$ to $10.89 \pm 2.34$ $\mathrm{ml}(P<0.01)$, respectively, while Qmax increased from 9.83 \pm 2.14 to $18.15 \pm 2.33 \mathrm{ml} / \mathrm{s}(P<0.01)$. The mean prostate volume (PV) decreased from $82.61 \pm 23.71 \mathrm{ml}$ to $32.78 \pm$ $3.54 \mathrm{ml}$ (with a mean reduction of $60.31 \%, P<0.01$ ). There was no significant difference in prostatic specific antigen (PSA) and international index of erectile function (IIEF) between pre-PAE and post-PAE. One patient had major event of skin necrosis of scrotal and perineal area $(1.5 \%)$. Recurrence of LUTS was noted in two patients $(2.9 \%)$.
\end{abstract}

Conclusions: PAE with Bletilla striata will provide evidence on the effectiveness and safety in treatment of LUTS of $\mathrm{BPH}$. However, more studies are needed to assess the long-term outcomes and potential complications.

\section{Keywords}

Benign prostatic hyperplasia, Lower urinary tract symptoms, Prostatic artery embolization, Bletilla striata

\section{Introduction}

Benign prostatic hyperplasia (BPH) is one of the most common problems in aging men, affecting over $50 \%$ of men older than 60 years in China [1]. Lower urinary tract symptoms (LUTS) resulting from BPH can spoil quality of life by impeding normal activities and causing complications such as acute urinary retention, urinary tract infection or even chronic renal failure. Medical therapy is usually the first-line treatment to symptomatic BPH [1]. However, the efficacy of drugs like alpha-blockers or 5-alpha reductase inhibitor is not only limited but also a time-consuming process. As disease progresses more invasive treatment options have to be taken into consideration. Transurethral resection of the prostate (TURP) remains the standard treatment in patients with moderate to severe LUTS. TURP, however, is limited to prostates smaller than $80 \mathrm{ml}$, the procedure is also associated with a substantial complication rate such as uroclepsia (1.1 2.2\%), bladder neck contracture (4\%), urethrostenosis (3.8\%), retrograde ejaculation (65\% 70\%) and sexual dysfunction (6.5\%) etc. [1]. The necessity for surgical revision is as high as $14.7 \%$ after TURP [2]. Furthermore, bleeding requiring transfusions (2 8.6\%)

Citation: Zhou X, Zhou S, Zhan P, Zhang J (2018) Prostatic Artery Embolisation with Bletilla Striata Particles in Treatment of Moderate to Severe Lower Urinary Tract Symptoms (LUTS) Resulted from Benign Prostatic Hyperplasia (BPH). Int Arch Nurs Health Care 4:106. doi.org/10.23937/24695823/15100106

Accepted: November 15, 2018: Published: November 17, 2018

Copyright: (C) 2018 Zhou X, et al. This is an open-access article distributed under the terms of the Creative Commons Attribution License, which permits unrestricted use, distribution, and reproduction in any medium, provided the original author and source are credited. 
and transurethral resection syndrome (2\%) represent potentially serious threats to elderly and frail patients [1-4]. Consequently, the development of new minimally invasive modalities for treatment of $\mathrm{BPH}$ refractory to medical therapy has become a hot research topic.

More recently, prostatic artery embolization (PAE) has showed promising results in animal experiments and clinical practice in relieving LUTS in patients with $\mathrm{BPH}$ [5]. The selection of embolic agents plays a crucial role in either treatment of $\mathrm{BPH}$ or in prevention of its recurrence, because different embolic agents and different particle size show different benefits. However, the optimal embolic agent and its size remain controversial [6-8]. We are inspired by the fact that satisfactory results of arterial embolization with Bletilla striata in solid benign or malignancy (such as hepatocellular carcinoma, renal carcinoma, uterus tumor, etc.) were achieved in last two decades [9-12]. In the present study, we report the results of PAE with Bletilla striata particles 150-200 $\mu \mathrm{m}$ in size as a primary treatment in 75 patients with severe LUTS after failure of medical treatment; all patients were unsuitable for surgery.

\section{Methods and Patient Selection}

In this clinical study, seventy-five patients with severe LUTS resulted from BPH will be analyzed 12 months of follow-up. This study was approved by the ethics committee of the hospital, and all patients signed a consent form to participate in the study. Patients with severe LUTS due to BPH refractory to medical treatment for at least 6-month underwent PAE using Bletilla striata particles from June 2013 to June 2018 at our Hospital. The mean age of patients was 75 years (range from 67 to 84 years). The base line data of these patients were summarized in Table 1. Inclusion criteria of PAE were symptomatic LUTS due to $\mathrm{BPH}$, age more than or equal to 50 years, international prostate symptom score (IPSS) $\geq 18$ and or quality of life $(\mathrm{QOL}) \geq 4$, peak urinary flow (Qmax) $<12 \mathrm{ml} / \mathrm{s}$, prostate volume $(\mathrm{PV})>40 \mathrm{ml}$, prostate serum antigen (PSA) was normal limit and/or patients with severe heart disease. Exclusion criteria were suspected prostate malignancy, neurogenic bladder dysfunction, serious coagulation disorders, large or multi bladder stones $(>2 \mathrm{~cm})$, active urinary tract infection, serious chronic renal failure and previous surgery of $\mathrm{BPH}$.

Efficacy was assessed by primary outcomes and sec-

Table 1: The clinical baseline data before PAE.

\begin{tabular}{|l|l|l|}
\hline Parameter & Values mean \pm SD & Range \\
\hline Age (year) & $75.0 \pm 1.25$ & $67.0-84.0$ \\
\hline IPSS (point) & $25.86 \pm 3.53$ & $19.21-33.6$ \\
\hline QOL score & $4.56 \pm 0.61$ & $4.3-5.9$ \\
\hline Qmax (ml/s) & $9.83 \pm 2.14$ & $6.1-12.0$ \\
\hline PV $(\mathrm{ml})$ & $82.61 \pm 23.71$ & $56.7-125.1$ \\
\hline PVR $(\mathrm{ml})$ & $131.52 \pm 19.13$ & $75.6-164.5$ \\
\hline IIEF $($ point) & $13.48 \pm 2.71$ & $11.9-14.3$ \\
\hline PSA $(\mathrm{ng} / \mathrm{ml})$ & $4.00 \pm 2.50$ & $2.6-8.1$ \\
\hline
\end{tabular}

ondary outcomes before and at $1,3,6$, and 12 months after the procedure. The former included IPSS, QOL and international index of erectile function (IIEF), and the latter was Qmax, PRV and PV etc. The curative outcomes are judged by following three reference standards, Excellence: (1) IPSS $\leq 7, Q \mathrm{QOL} \leq 1,(2) \mathrm{PV}$ is reduced to less than $60 \%$, (3) Qmax $\geq 18 \mathrm{ml} / \mathrm{s}$. More than two items are considered to meet standards. Improvement: (1) IPSS $\leq$ $13, \mathrm{QOL} \leq 4-6$, (2) PV is reduced to less than $50 \%$, (3) Qmax $\geq 12 \mathrm{ml} / \mathrm{s}$. More than one item is considered to meet standards. Ineffectiveness: no effective standard.

\section{Preparation of Bletilla striata angioembolus}

The original material was the stem tubers of Bletilla striata. The method of preparation was described previously as described by Zheng, et al. [9] and provided by Pharmacological Institute of the Hospital [13]. A dose of $1.0 \mathrm{mg}$ Bletilla striata microparticles $150-200 \mathrm{~mm}$ was suspended in $1 \mathrm{ml} 0.9 \% \mathrm{NaCl}$ for ten minutes before administration.

\section{Embolization technique}

Prophylactic antibiotic (ceftriaxone, $1 \mathrm{~g} / \mathrm{d}$, Roche Pharmaceutical Ltd., Shanghai, China) was routinely administrated thirty minutes before procedure and continued for three days following PAE. After local anesthesia was achieved, femoral arterial access was gained percutaneously with a 5-F introducer sheath (Radifocus, Terumo, Tokyo, Japan) with Seldinger's technique. Pelvic angiography (Allura Xper FD20, Philips, Netherlands) was performed with a 5-F pigtail catheter, then a 5-F Cobra catheter (Radifocus, Terumo, Tokyo, Japan) was inserted into the internal iliac artery selectively to evaluate the branches and prostatic artery (PA) which was identified with DSA (Figure 1). Superselective catheterization of the inferior vesicle arteries or other branch

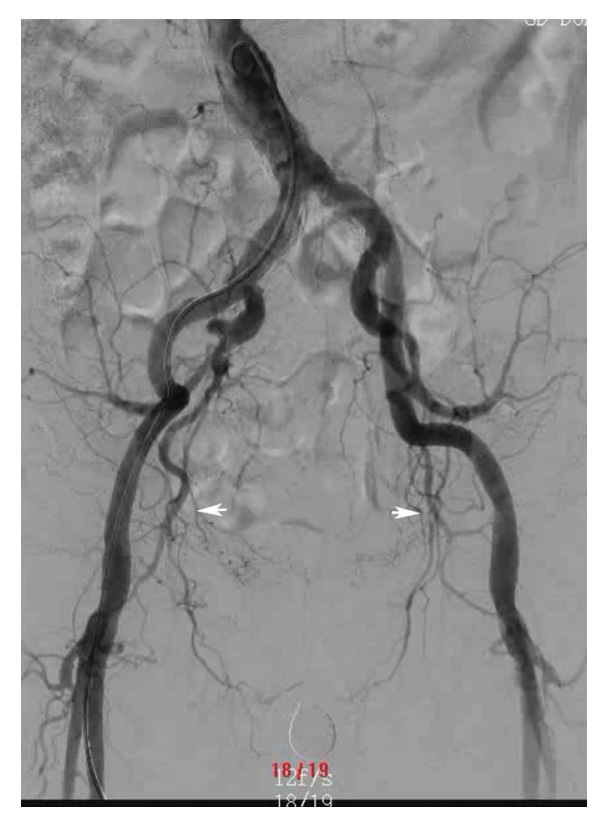

Figure 1: Pelvic angiogram showed the external and internal iliac arteries, their branches, and the prostate artery (white arrow). 

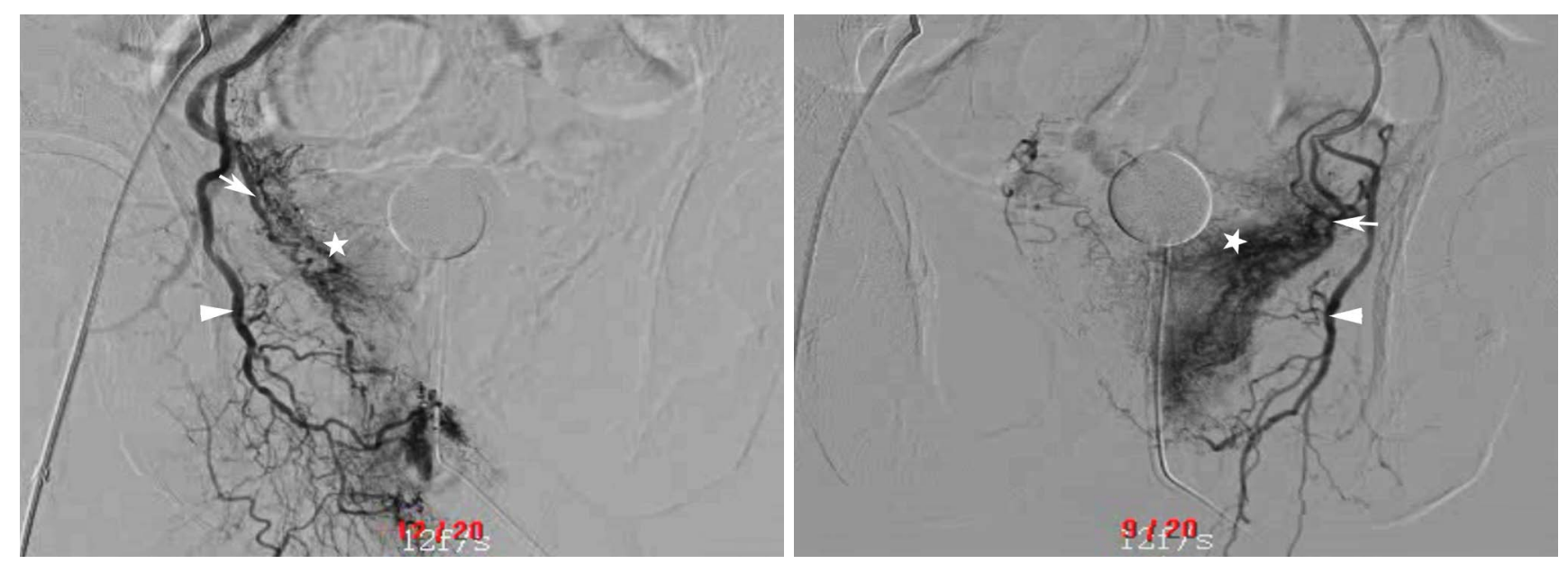

Figure 2: Superselective prostatic artery angiogram showed that bilateral prostate arteries (white arrows) issued from the gluteal-pudendal trunks (white arrow heads) (A right side, B left side). The white arrows indicated the enlarged corkscrew pattern of intra-prostate arteriola and the five stars demonstrated a prominent prostatic blush consistent with benign prostatic hyperplasia.
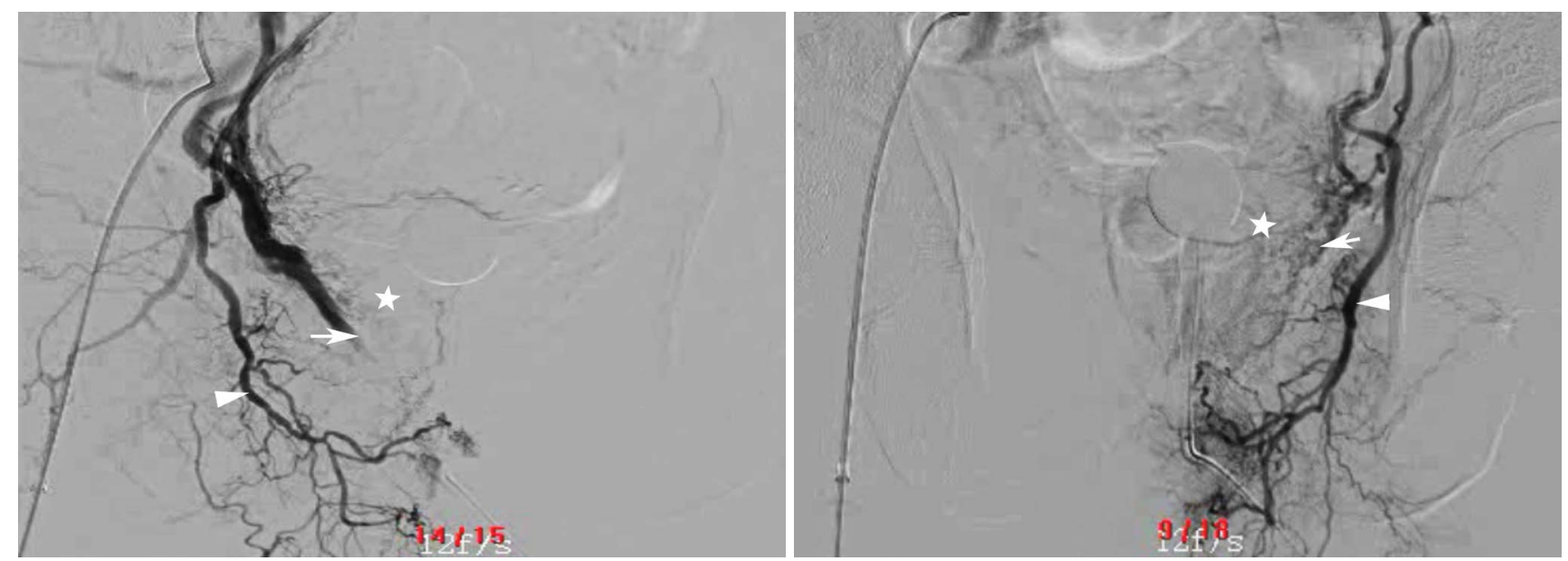

Figure 3: Superselective postembolization internal iliac arteriogram showed absent flow (white arrows) to the enlarged prostate (A right side, $\mathbf{B}$ left side) and the five stars demonstrated the vascular staining in the prostate disappeared greatly. Note the preservation of the internal pudendal artery (white arrow heads).

was then performed using a coaxial 2.8-F microcatheter (Progreat, Terumo, Tokyo, Japan) and angiography was performed by manually injecting 3-5 $\mathrm{ml}$ of contrast medium (lopromide 370, Bayer Pharmaceutical Ltd., Berlin, Germany) to ensure that the tip of the microcatheter was inside or at the ostium of the prostatic arteries (Figures $2 \mathrm{~A}$ and Figure 2B). Each $1 \mathrm{ml}$ vial of Bletilla striata microparticles was diluted in a $20-40 \mathrm{~mL}$ solution of nonionic contrast medium. The mixture was slowly injected under fluoroscopic guidance. Embolization of the prostate arteries was performed to stasis without reflux of the mixture to untargeted arteries (Figure $3 \mathrm{~A}$ and Figure 3B). After the procedure, angiography was performed using the high pressure injector, with the $5-\mathrm{F}$ catheter at the anterior branch of the internal iliac artery to check for any further blood supply to the prostate. Embolization was then performed on the other side using the same technique.

\section{Statistical analysis}

The quantitative variables were expressed as mean \pm standard deviation (SD) and range, while the categorical data were shown as count and percentage. A Student's $t$-test for paired samples was used when appropriate. A value of $P<0.01$ was considered to be statistically significant.

\section{Results}

PAE was successfully performed in 68 of 75 patients $(90.7 \%)$. Seven technical failures $(9.3 \%)$ were attributed to severe tortuosity and atherosclerosis of the iliac arteries. Bilateral PAE was successful in 61 (89.7\%) patients; while the remaining seven $(10.3 \%)$ patients were performed unilateral PAE because of another side internal iliac artery stenosis PA. Mean operative time was $120 \mathrm{~min}$ (range 100-200 min).

Follow-up data of sixty-eight patients were available, who were observed for a mean of 14 months (range 4-40 months). The total effective rate at 1, 3, 6, 12 months was $100 \%$ (68/68), 100\% (68/68), 95.5\% $(65 / 68)$, and $95.5 \%(65 / 68)$, respectively. As shown in 
Table 2: Clinical data prior to PAE using Bletilla striata particles and each to follow-up evaluation.

\begin{tabular}{|c|c|c|c|c|c|c|c|}
\hline \multirow{2}{*}{ Variable } & \multirow{2}{*}{$\begin{array}{l}\text { Before } \\
(n=72)\end{array}$} & \multicolumn{4}{|l|}{ After (month) } & \multirow[b]{2}{*}{$F$} & \multirow[b]{2}{*}{$\boldsymbol{P}$} \\
\hline & & $1(n=68)$ & $3(n=68)$ & $6(n=68)$ & $12(n=68)$ & & \\
\hline IPSS (point) & $25.86 \pm 3.53$ & $21.15 \pm 3.32$ & $16.23 \pm 3.80$ & $12.03 \pm 1.77$ & $6.52 \pm 1.51$ & 113.42 & 0.000 \\
\hline QOL score & $4.56 \pm 0.61$ & $3.73 \pm 0.53$ & $2.81 \pm 0.45$ & $2.45 \pm 0.39$ & $1.26 \pm 0.43$ & 95.13 & 0.001 \\
\hline IIEF (point) & $13.48 \pm 2.71$ & $13.53 \pm 2.63$ & $13.91 \pm 2.71$ & $14.00 \pm 2.67$ & $13.65 \pm 2.17$ & 8.79 & 0.062 \\
\hline Qmax (ml/s) & $9.83 \pm 2.14$ & $13.26 \pm 1.95$ & $17.01 \pm 2.32$ & $17.52 \pm 2.13$ & $18.15 \pm 2.33$ & 112.18 & 0.000 \\
\hline $\mathrm{PV}(\mathrm{ml})$ & $82.61 \pm 23.71$ & $73.51 \pm 12.58$ & $53.58 \pm 9.11$ & $43.64 \pm 7.80$ & $32.78 \pm 3.54$ & 106.48 & 0.000 \\
\hline PRV (ml) & $131.52 \pm 19.13$ & $43.25 \pm 7.68$ & $30.63 \pm 4.11$ & $13.25 \pm 2.93$ & $10.89 \pm 2.34$ & 112.41 & 0.000 \\
\hline
\end{tabular}

IPSS: International prostate symptom score; QOL: Quality of life; IIEF: International index of erectile function; Qmax: Peak urinary flow rate; PV: Prostate volume; PRV: Postvoid residual urine.

Table 2, LUTS resulted from BPH was improved effectively. At 12-month follow-up of sixty-eight patients, the mean IPSS decreased from $25.86 \pm 3.53$ points to $6.52 \pm$ 1.51 points $(P<0.01)$, mean QOL decreased from $4.56 \pm$ 0.61 points to $1.26 \pm 0.43$ points $(P<0.01)$, while mean Qmax increased from $9.83 \pm 2.14$ to $18.15 \pm 2.33 \mathrm{ml} / \mathrm{s}$ $(P<0.01)$. Mean PVR decreased from $131.52 \pm 19.13 \mathrm{ml}$ to $10.89 \pm 2.34 \mathrm{ml}(P<0.01)$, and mean $\mathrm{PV}$ decreased from $82.61 \pm 23.71 \mathrm{ml}$ to $32.78 \pm 3.54 \mathrm{ml}$ (with a mean reduction of $60.31 \%, P<0.01)$. There were no significant differences $(P=0.062)$ in IIEF scores before and 12 months after PAE.

The serum total PSA values before PAE were normal. The mean serum total PSA increased $24 \mathrm{~h}$ after embolization (from $4.00 \pm 2.50 \mathrm{ng} / \mathrm{ml}$ to $87.50 \pm 45.00 \mathrm{ng}$ / $\mathrm{mL}$ ) and peaked at next 24 to $48 \mathrm{~h}$. Then PSA began to decrease one week following PAE and fell to the normal range after one month. In the follow-up of 12 months, the mean PSA sustained in normal limit.

The complication rate was $14.7 \%(10 / 68)$ in our series. Minor complication accounted for $90 \%$ (9/10), including four cases of transient hematuria (5.8\%) and five cases of rectal bleeding accompanied perineal region pain (7.4\%). All these minor complications disappeared during the first one or two weeks. However, we did encounter one patient with serious skin necrosis of scrotal and perineal area (1.5\%) resulted from probably untargeted embolization. The patient recovered after debridement and wound sutures without obvious sequela. No prostate infection or abscess were found in our study. There were two recurrences of LUTS after 10 months on unilateral PAE (recurrent rate was $2.9 \%$ ).

\section{Discussion}

Theoretically, the major rationale of PAE for $\mathrm{BPH}$ is based on two aspects. On one hand, although BPH aetiology remains fully understood, several mechanisms have been proposed to be involved in the pathogenesis and progression of $\mathrm{BPH}$. Accumulating evidence indicates that the development and progression of $\mathrm{BPH}$ relies on angiogenesis to obtain adequate oxygen and nutrients from the nearby blood vessels by simple passive diffusion, which promotes prostatic cell proliferation and inhibits apoptosis, leading to prostatic enlargement. Lin, et al. [14] have showed that the hypoxia-in- ducible factor- $1 \alpha$ (HIF-1 $\alpha$ ) signaling pathway promotes the process of angiogenesis, contributing to the growth and progression of $\mathrm{BPH}$ in animal models. It has been proposed that neovascularization began in $\mathrm{BPH}$ and that it kept progressing in a stepwise manner in the subsequent proliferative premalignant and malignant stages. Consequently, BPH was sometimes referred as to "benign tumor" because of resembling solid tumor growth pattern [1,3]. Shen and Tao [15] have also demonstrated that the blood flow velocity values are much faster, the inner diameters of the vessels are much wider, and the vascular tone is much higher in BPH patients than those of normal prostate. PAE was able to cause extensive and complete intra-prostatic ischemia and anoxia, directly resulting in necrosis, sclerosis, apoptosis, and subsequent shrinkage, from which LUTS is helpfully relieved. On the other hand, most experts consider androgens (testosterone and related hormones) to play a permissive role in the development of $\mathrm{BPH}$. This means that androgens must be present for $\mathrm{BPH}$ to occur, but do not necessarily directly cause the condition. This is supported by evidence suggesting that castrated boys (for example eunuchs in the Qing dynasty, China) do not develop BPH when they age [16]. Dihydrotestosterone (DHT), a metabolite of testosterone, is a critical mediator of prostatic growth. It binds to nuclear androgen receptors and signals the transcription of growth factors that are mitogenic to the epithelial and stromal cells, promoting prostate cell proliferation $[16,17]$. If the blood supply to the prostate is terminated by means of PAE and the periurethral area of the transitional zone (site of the primary BPH nodule) is devascularized, functional androgen-signaling axis would be interrupted by blocking the transport of testosterone and DHT to the prostate. The testosterone and converted DHT would be at extremely low levels in the gland tissue, consequently, prostatic cell apoptosis and subsequent therapeutic responses can be achieved, similarly to other clinical therapies such as finasteride and surgical/chemical castration.

Clinically, PAE was first described in 1977 by Bischoff, et al. [18] as a treatment option in intractable prostatogenic haemorrhage. DeMeritt, et al. first reported that they successfully managed one BPH patient with persistent hematuria using superselective transarterial polyvinyl alcohol (PVA) of 150-250 $\mu \mathrm{m}$ embolization 
in 2000 [19]. First intentional treatment of $B P H$ by PAE with 300-500 $\mu \mathrm{m}$ PVA in diameter was published in 2010 by Carnevale and his colleagues [2]. Pisco, et al. [20] reported that PAE (100-200 $\mu \mathrm{m}$ non-spherical PVC) was technically successful in 250 of the 255 (98\%) selected patients. Promising short-term and medium-term results could be shown for patients with symptomatic $\mathrm{BPH}$, refractory to medical treatment. More recently, Wang, et al. [21] have noted in their study that particles with $50 \mu \mathrm{m}$ in size may penetrate into the periurethral region of the prostate, with a better result than that of particles $\geq 100 \mu \mathrm{m}$ in size. However, untargeted embolization and injury of the urethral wall should be concerned using the small sized particles. In China, Lin, et al. [22] reported that PAE was performed in 132 symptomatic patients using Chinese traditional medicine Zedoary Oil and Brucea Javanica Oil as embolic materials. Medium-term clinical outcomes were satisfactory, however, $13.6 \%$ of these patients were needed to receive TURP for recurrent LUTS in a long-term follow-up. Gao, et al. [23] have compared the effectiveness of PAE (355$500 \mu \mathrm{m}$ PVA) with the reference standard of TURP in patients with LUTS related to $\mathrm{BPH}$ and have pointed out that the overall adverse event and complications in PAE group was much higher when compared with TURP group, showing no superiority over TURP treatments. Although PAE was shown to be a safe procedure with low morbidity in carefully selected patients, however, data concerning PAE has been criticized for different reasons: Small number of studies were described so far. Quality of the studies available was referred to be poor due to study type. Patients selections were unclear and some studies involved dropouts as well as statistical limitations [7]. The long-term outcome of these embolized prostates is still unknown. Revascularization after PAE may play a role in recurrent symptoms of LUTS and prostate re-growth. Carnevale, et al. [24] have reported the initial two patients presented signs of de novo prostate growth as measured through MRI after maintained clinical benefit for 4 years. Consequently, embolic material does play a key role in $\mathrm{PAE}$, however, what type and what size of PAE embolic microparticle used remains controversial.

Bletilla striata, known is a species of orchid native to China and Southeast Asia, often used in Chinese traditional medicine for treating problems such as alimentary canal mucosal damage, ulcers, bleeding and burns. In the last two decades, Bletilla striata used as arterial embolus, for treatment of unresectable malignancies such hepatic carcinoma, renal carcinoma, bone tumor, myoma of uterus etc, has been reported with encouraging results [25], especially for inhibiting arterial collaterals of liver tumor and recurrence of hepatocellular carcinoma [9]. The mechanisms of embolization caused by Bletilla striata are as follows: (1) Blatilla striata particles have an adherent function and can expand slowly in blood flow, leading to mechanical blockade of vessels.
Meanwhile, it cannot be absorbed by body tissues [26]. (2) Bletilla striata particles spur hemostatic modes of action by forming a visible particle/blood aggregate as a physical barrier that gives rise to hemostasis. The hemostatic results of 350-250 $\mu \mathrm{m}$ particles were found to be the most efficient [27]. (3) Bletilla striata has the activity anti-inflammatory. More interestingly, it was reported to be able to play dual roles in inflammation, maintaining an appropriate inflammatory response that could benefit for wound recover [28]. Moreover, Bletilla striata shows potent antibacterial activities against not only fungal strains but also Gram-positive bacterial strains including Methicillin-resistant Staphylococcus aureus (MRSA) [26]. (4) Bletilla striata displays interesting anticancer activity by means of production of reactive oxygen species (ROS) to lead to cancer cell apoptosis and/ or inhibition of the tumor necrosis factor alpha (TNF- $\alpha$ ) level and the elevation of the epidermal growth factor (EGF) secretion $[29,30]$.

In our experience, PAE with Bletilla striata particles has been demonstrated to be satisfactory for the relief of LUTS in the study after medium-term follow-up. The method provided a low rate recurrence (2.9\%), a low risk of postoperative complications (14.7\%). There were no cases of prostate abscess, blood transfusion, urinary incontinence or sexual dysfunction.

The feasibility of a safe and effective PAE depends on many factors, including the characteristics of $\mathrm{BPH}$, body weight, habitus, age, increased atherosclerosis and the ability and experience of the operating physicians, and most importantly the embolic materials used. Although our technical success rate was only $90.7 \%$, this technique of PAE by Bletilla striata particles offered some advantages: First, instead of PVA, Zedoary Oil and Brucea Javanica Oil used as mentioned above [22,23], Bletilla striata was based on empiricism of embolization of benign or malignancy in the last two decades [9-12,30]. It has the ability of expansion and promotion of thrombin and produce extensive and permanent vascular embolization, while it cannot be absorbed by body tissues. After embolization, prostate necrosis and shrinkage were significant with less collateral circulation that formed later [9-12]. Second, the polysaccharide component of Bletilla striata was a wide spectrum antitumor element that may inhibit tumor occurrence and development by inhibiting angiogenesis and anti-mitotic effect [30]. To date Bletilla striata was the unique embolic agent that had a dual role of embolization and inhibition of tumor collateral circulation. Recurrence of LUTS after PAE varied from $8.3 \%$ to $13.6 \%[21,22]$. We had two recurrent patients with unilateral PAE, and that may be attributed to development of collateral circulation. Third, it has been reported that $43-98 \%$ of specimens from BPH had acute and chronic prostate inflammation, increasing in IPSS, and greater risk of complications [30]. Unlike other emboluses, Bletilla striata had unique anti-inflammatory properties that could further improved symptoms of LUTS. 
Prostate infection and/or abscess after PAE are rare complications that have been estimated to occur at a rate of $1.9 \%$ [23]. However, our study showed that no prostate abscess occurred after embolization, this may be related to Bletilla striata antibiotic activity. And the last, it has also been proved that Bletilla striata was pyrogen-free and had good tissue biocompatibility and no adverse effects, such as hepatotoxicity and renal toxicity in in vitro and in vivo, indicating their promising potential for use as a highly biocompatible embolic agent $[11,12,26]$. Moreover, Bletilla striata is rich in nature and very economical in use.

Although PAE with Bletilla striata was efficacious, however, the complications were related to the risks associated with inadvertent or untargeted ischemia affecting the bladder, rectum, anus and/or corpus cavernosum, resulting in transient haematuria (5.7\%), transient rectal bleeding (7.4\%) and perineal pain. In the study, one patient had serious necrosis in scrotal and perineal region (1.5\%) resulted from nontargeted embolization, which may be explained as a technical failure. This happened in the first ten patients and he recovered after debridement. Although post-embolization complications observed in this study were $14.7 \%$, but mostly of minor importance, in comparison with the findings of others [19-22].

\section{Conclusion}

The present study showed that PAE with Bletilla striata particles is efficacious, safe and economical to patients with LUTS due to BPH. It is easy to use and has many advantages. However, the advantages of PAE must be weighed against the risk of technical and clinical failures. Future studies with larger sample sizes and longer follow-up time are needed to explore the mechanism of apoptosis of BPH induced by Bletilla striata and also needed to assess long-term outcomes and potential complication with Bletilla striata particles for PAE.

\section{Disclosure Statement}

The authors have no conflicts of interest regarding the publication of this paper.

\section{Funding}

Thanks are due to the following foundations for their grants to perform this study: Dongguan Science and Technology Bureau (No: 201450715000167).

\section{References}

1. Ye G, Zhou XL (2017) Clinical advances of the prostatic artery embolization in treatment of benign prostatic hyperplasia. Chin J Inter Rad 5: 34-38.

2. Carnevale FC, Antunes AA, da Motta Leal Filho JM, de OIiveira Cerri LM, Baroni RH, et al. (2010) Prostatic artery embolization as a primary treatment for benign prostatic hyperplasia: Preliminary results in two patients. Cardiovasc Intervent Radiol 33: 355-361.

3. Sun F, Sánchez FM, Crisóstomo V, Díaz-Güemes I,
López-Sánchez C, et al. (2011) Transarterial prostatic embolization: Initial experience in a canine model. AJR Am J Roentgenol 197: 495-501.

4. Madersbacher S, Marberger M (1999) Is transurethral resection of the prostate still justified? BJU Int 83: 227-237.

5. Sun F (2016) Prostatic artery embolization: A potential treatment option for localized prostate cancer. Clin Oncol 1: 1008

6. Schreuder SM, Scholtens AE, Reekers JA, Bipat S (2014) The role of prostatic arterial embolization in patients with benign prostatic hyperplasia: A systematic review. Cardiovasc Intervent Radiol 37: 1198-1219.

7. Kaplan SA (2017) Prostate arterial embolization is a viable option for treating symptoms of benign prostatic hyperplasia: Con. J Urol 198: 9-11.

8. Li Q, Duan F, Wang MQ, Zhang GD, Yuan K (2015) Prostatic arterial embolization with small sized particles for the treatment of lower urinary tract symptoms due to large benign prostatic hyperplasia: Preliminary results. Chin Med J (Engl) 128: 2072-2077.

9. Zheng $C$, Feng $G$, Liang $H$ (1998) Bletilla striata as a vascular embolizing agent in interventional treatment of primary hepatic carcinoma. Chin Med J (Engl) 111: 1060-1063.

10. Rammohan A, Sathyanesan J, Ramaswami S, Lakshmanan A, Senthil-Kumar P, et al. (2012) Embolization of liver tumors: Past, present and future. World J Radiol 4: 405-412.

11. Luo S, Song S, Zheng C, Wang Y, Xia X, et al. (2015) Biocompatibility of Bletilla striata microspheres as a novel embolic agent. Evid Based Complement Alternat Med 2015: 840896 .

12. Luo SH, Song SL, Zheng CS, Li WY, Wang Y, et al. (2017) Embolic effects of Bletilla striata microspheres in renal artery and transplanted VX2 liver tumor model in rabbits. Chin J Integr Med.

13. Zhou XL, Zhou SJ, Zhan P, Ye G, Zhang J (2017) Clinical study of prostatic arterial embolization jointly with Bletilla Striata for the treatment of benign prostatic hyperplasia. Chin J Inter Rad 5: 89-93.

14. Lin J, Zhou J, Xu W, Hong Z, Peng J (2014) Qianliening capsule inhibits benign prostatic hyperplasia angiogenesis via the HIF-1a signaling pathway. Exp Ther Med 8: 118124.

15. Shen J, Cheng T (2015) Changes of blood supply to benign prostatic hyperplasia and its clinical significance. J Shanghai Jiaotong Med Sci 35: 414-416.

16. Wu CP, Gu FL (1991) The prostate in eunuchs. Prog Clin Biol Res 370: 249-255.

17. Xu D, Wang X, Jiang C, Ruan Y, Xia S, et al. (2017) The androgen receptor plays different roles in macrophage-induced proliferation in prostate stromal cells between transitional and peripheral zones of benign prostatic hypertrophy. EXCLI J 16: 939-948.

18. Bischoff W, Goerttler U (1977) Successful intra-arterial embolization of bleeding carcinoma of the prostate. Urologe $A$ 16: 99-102.

19. DeMeritt JS, Elmasri FF, Esposito MP, Rosenberg GS (2000) Relief of benign prostatic hyperplasia-related bladder outlet obstruction after transarterial polyvinyl alcohol prostate embolization. J Vasc Interv Radiol 11: 767-770.

20. Pisco JM, Rio Tinto H, Campos Pinheiro L, Bilhim T, Duarte $\mathrm{M}$, et al. (2013) Embolisation of prostatic arteries as 
treatment of moderate to severe lower urinary symptoms (LUTS) secondary to benign hyperplasia: Results of shortand mid-term follow-up. Eur Radiol 23: 2561-2572.

21. Wang MQ, Guo LP, Zhang GD, Yuan K, Li K, et al. (2015) Prostatic arterial embolization for the treatment of lower urinary tract symptoms due to large $(>80 \mathrm{~mL})$ benign prostatic hyperplasia: Results of midterm follow-up from Chinese population. BMC Urol 15: 33.

22. Lin JZ (2006) The clinical research of the oil of Zedoary Turmer and Seed of Brucea in treatment of benign prostatic heperplasia by blood vessel interventional therapy. University of Guangzhou Traditional Chinese Medicine, Guangzhou.

23. Gao YA, Huang Y, Zhang R, Yang YD, Zhang Q, et al. (2014) Benign prostatic hyperplasia: Prostatic arterial embolization versus transurethral resection of the prostate--a prospective, randomized, and controlled clinical trial. Radiology 270: 920-928.

24. Carnevale FC, da Motta-Leal-Filho JM, Antunes AA, Baroni $\mathrm{RH}$, Freire GC, et al. (2011) Midterm follow-up after prostate embolization in two patients with benign prostatic hyperplasia. Cardiovasc Intervent Radiol 34: 1330-1333.
25. Song JL, Wang L, Han FJ (2013) The retrospective analysis of Rhizoma Bletillae used as an antitumor medicine. Inform Trad Chin Med 30: 148-150.

26. He X, Wang X, Fang J, Zhao Z, Huang L, et al. (2017) Bletilla striata: Medicinal uses, phytochemistry and pharmacological activities. J Ethnopharmacol 195: 20-38.

27. Zhang C, Zeng R, Liao Z, Fu C, Luo H, et al. (2017) Bletilla striata micron particles function as a hemostatic agent by promoting rapid blood aggregation. Evid Based Complement Alternat Med 2017: 5820405.

28. Yue L, Wang W, Wang Y, Du T, Shen W, et al. (2016) Bletilla striata polysaccharide inhibits angiotensin II-induced ROS and inflammation via NOX4 and TLR2 pathways. Int $\mathrm{J}$ Biol Macromol 89: 376-388.

29. Sun A, Liu J, Pang S, Lin J, Xu R (2016) Two novel phenanthraquinones with anti-cancer activity isolated from Bletilla striata. Bioorg Med Chem Lett 26: 2375-2379.

30. Nickel JC, Roehrborn CG, O'Leary MP, Bostwick DG, Somerville MC, et al. (2008) The relationship between prostate inflammation and lower urinary tract symptoms: Examination of baseline data from the REDUCE trial. Eur Urol 54: 1379-1384. 